\title{
PHYSIOLOGICAL MECHANISMS OF EVOLVED DESICCATION RESISTANCE IN DROSOPHILA MELANOGASTER
}

\author{
ALLEN G. GIBBS*, ADAM K. CHIPPINDALE† AND MICHAEL R. ROSE \\ Department of Ecology and Evolutionary Biology, University of California, Irvine, CA 92697, USA
}

Accepted 9 April 1997

\begin{abstract}
Summary
We investigated physiological characters associated with water balance in laboratory populations of Drosophila melanogaster selected for resistance to desiccating conditions for over 100 generations. Five replicate, outbred, desiccation-selected (D) populations were compared with their control (C) populations. Water loss rates of female $D$ flies were approximately $40 \%$ lower than those of $\mathrm{C}$ females. Although excretory water loss was reduced in desiccation-selected flies, it comprised less than $10 \%$ of total water loss, indicating that the $D$ populations have evolved reduced cuticular and/or respiratory water

slightly higher temperatures, possibly contributing to reduced water loss rates. Desiccation-selected flies contained approximately $30 \%$ more bulk water than controls, as well as more glycogen. However, total metabolic water stores did not differ between the stocks owing to higher lipid levels in the $\mathrm{C}$ populations. The ability to tolerate water loss, as measured by water content at the time of death, did not differ between $D$ and $C$ flies. Thus, evolution of increased desiccation resistance has occurred by multiple physiological mechanisms, but some potential adaptive differences have not evolved.
\end{abstract} loss rates. Total surface lipid amounts did not differ between the $\mathrm{C}$ and $\mathrm{D}$ flies. Cuticular hydrocarbons from $\mathrm{D}$ flies were longer than those from $\mathrm{C}$ flies and melted at

Key words: desiccation, fruit fly, Drosophila melanogaster, water balance, evolution, cuticular lipid, laboratory selection.

\section{Introduction}

One of the most important environmental stresses facing insects in nature is desiccation. The small size (and therefore large ratio of surface area to volume) of terrestrial arthropods makes them particularly susceptible to dehydration. Numerous comparative studies have shown that insects from warmer, drier environments exhibit a variety of apparently adaptive differences in water balance, e.g. reduced cuticular permeabilities, reduced excretory water loss and differences in the quantity and composition of cuticular lipids (reviewed by Hadley, 1994a).

Comparative studies of adaptation can be strengthened by phylogenetically based analyses of the pattern of evolution (Harvey and Pagel, 1991; Garland and Adolph, 1994). However, reliable information about the phylogenetic relationships of organisms is often unavailable. Even if this information is available, several other factors (e.g. genetic correlations, linkage disequilibrium, pleiotropy, parental effects, genotype-by-environment interactions) can undermine one's confidence in the adaptive significance of a given trait (Lauder et al. 1993; Leroi et al. 1994). In cases in which a trait does have current adaptive value (as indicated, for example, by field studies of selection; see Endler, 1986), it is impossible to determine precisely which selective factors influenced the evolution of this trait. Did it arise as a result of the same selective forces now operating in nature, has a trait evolved for a different purpose been recruited for a new function, or is it a product of stochastic processes? If a trait has not evolved in response to selection, why not? Is its evolution constrained by the pleiotropic effects of genetic correlations with other characters, or has there simply been insufficient genetic variation or selection to affect the trait?

Comparative studies are inherently correlative, attempting to reconstruct the history of selection from its endpoints. A complementary approach to the comparative method is to study the evolutionary process in the laboratory. The laboratory evolution approach avoids many of the potential pitfalls of interspecific comparative studies, since the selective regime is known and can be manipulated by the investigator (Rose et al. 1990, 1996; Garland and Carter, 1994). Numerous researchers have shown that organisms respond to selection for increased resistance to environmental stresses, e.g. temperature (Bennett et al. 1990; Huey et al. 1991; Lenski and Bennett, 1993), starvation (Rose et al. 1992) and urea (Gupta et al. 1995; Joshi et al. 1996). However, the genetic or physiological basis of evolved stress resistance is frequently unclear. Polygenic and oligogenic variation have each been observed in response to

\footnotetext{
*e-mail: agibbs@uci.edu.

$\dagger$ Present address: Department of Biology, NS IV, University of California, Santa Cruz, CA 95064, USA.
} 


\section{A. G. Gibbs, A. K. Chippindale and M. R. Rose}

selection for pesticide resistance (Crow, 1957; McKenzie and Clarke, 1988). Hoffmann and Parsons (1989, 1991) have hypothesized that reduced metabolic rates constitute a generalized response to stress selection, although this proposal has been disputed by Djawdan et al. (1997).

Several investigators have shown that the desiccation resistance of Drosophila melanogaster can be increased by selection (Hoffmann and Parsons, 1989; Rose et al. 1990, 1992; Blows and Hoffmann, 1993). Typical selection protocols involve exposing flies to dry air and using the longestsurviving individuals to propagate the next generation. From an organismal standpoint, there are only three mechanisms by which resistance to such an acute desiccation stress can be enhanced: rates of water loss can be reduced, flies can contain more water (as bulk water or metabolic water stores) or flies can tolerate the loss of greater amounts of water. Extreme examples of the last possibility are anhydrobiotic organisms, such as baker's yeast, brine shrimp embryos or seeds, which can survive for months or years in a dry state (Crowe et al. 1992). These mechanisms of desiccation resistance are not exclusive of one another.

Physiological studies of evolved mechanisms of desiccation resistance in Drosophila spp. have been limited and sometimes contradictory. Graves et al. (1992) found that desiccationselected populations of $D$. melanogaster contained more glycogen than controls, whereas Blows and Hoffmann (1993) found no correlation with potential metabolic water stores (glycogen and lipid) in selected lines of D. serrata. Instead Blows and Hoffmann (1993) observed that selection had resulted in reduced metabolic rates under desiccating conditions. Djawdan et al. (1997), using the same populations as Graves et al. (1992), found no differences in metabolic rates between desiccation-selected and control populations, when corrected for differences in metabolic reserves.

The conflicting results of selection experiments may be due to differences in species, selection protocols, the number of generations of selection, etc. In addition, each of these studies examined only one or a few components of water balance. We describe here experiments investigating the mechanistic basis of evolved desiccation resistance in five outbred populations of D. melanogaster selected for more than 100 generations. We measured the rate at which flies lost water. We examined several aspects of cuticular lipids: quantity, composition and physical properties. We determined how much water (both bulk and metabolic water) the flies contained, and we determined how much loss of overall body water the flies could tolerate. By constructing a complete water budget for flies from these populations, we were able to assess the relative contributions of these characters to overall desiccation resistance.

\section{Materials and methods}

\section{Drosophila melanogaster populations}

The origins and maintenance of the populations have been described previously (Rose et al. 1990, 1992). In short, five O- stocks $\left(\mathrm{O}_{1}-\mathrm{O}_{5}\right)$ were derived in 1980 from a large, outbred laboratory population, originally collected in Amherst, MA, USA, in 1975 (Rose, 1984). These were selected for postponed age of reproduction, resulting in greatly increased lifespan in these populations relative to five control $\left(\mathrm{B}_{1}-\mathrm{B}_{5}\right)$ populations. The O-stocks have been maintained as outbred populations on a 10 -week generation cycle since 1981. All stocks described here are maintained with constant light at $25^{\circ} \mathrm{C}$ on banana-molasses medium.

Service et al. (1985) found that the O populations exhibited greater resistance to a variety of environmental stresses, including desiccation, relative to five control populations maintained on the 2-week life cycle of the stock that was ancestral to both $\mathrm{O}$ populations and their controls. Service et al. (1988) then derived reverse selection lines from the $\mathrm{O}$ populations, these lines being handled in the same manner as the controls, with 2-week generations. In the course of more than 20 generations, starvation resistance and early fecundity responded to reverse selection, but desiccation resistance did not. In order to determine whether this lack of response to reverse selection was due to an exhaustion of genetic variation for desiccation resistance in the $\mathrm{O}$ populations, Rose et al. (1990) initiated selection for increased desiccation resistance in derivatives of $\mathrm{O}$ populations (designated $\mathrm{D}_{1}-\mathrm{D}_{5}$, one from each $\mathrm{O}$ population). A control (C) population was also founded from each $\mathrm{O}$ population at the same time. The five $\mathrm{C}$ populations have been maintained identically to the $D$ population, except for the absence of desiccation selection. All populations have been maintained as large outbred populations (approximately 1000 individuals per generation), in order to minimize the effects of inbreeding and genetic drift.

Since being founded in 1988, the C and D flies have been reared in vials for 2 weeks (including all pre-adult stages and 3-4 days as adults; Fig. 1). At 14 days from the egg stage, flies are transferred to population cages. The D cages contain approximately $150 \mathrm{~g}$ of fresh Drierite desiccant and are sealed with plastic wrap to maintain low humidity. The D flies are therefore subjected to both desiccation and the absence of food. In order to control for the effects of starvation, the $\mathrm{C}$ populations are reared in the same manner as the $\mathrm{D}$ population, but their cages are provided with a non-nutritive agar plate instead of desiccant. These flies are not subjected to desiccation selection, but do, however, undergo mild selection for starvation resistance. For a given D population, when approximately $80 \%$ of the flies have died, the desiccant is removed, and the flies are provided with food. Each C-stock is paired with its corresponding D-stock during selection; when a given stock $\mathrm{D}_{\mathrm{i}}(i=1-5)$ is given food, so is its corresponding $\mathrm{C}_{\mathrm{i}}$ population.

Our experiments were designed to provide as realistic a picture as possible of the water relationships of these flies at the time they entered selection. Thus, assays were performed when the flies were 14 days from the egg stage (3-4 days posteclosion), the age at which the D populations were subjected to desiccation selection (and the $\mathrm{C}$ populations were starved). For all assays, flies were reared for two generations on a 
Desiccation resistance in Drosophila melanogaster 1823

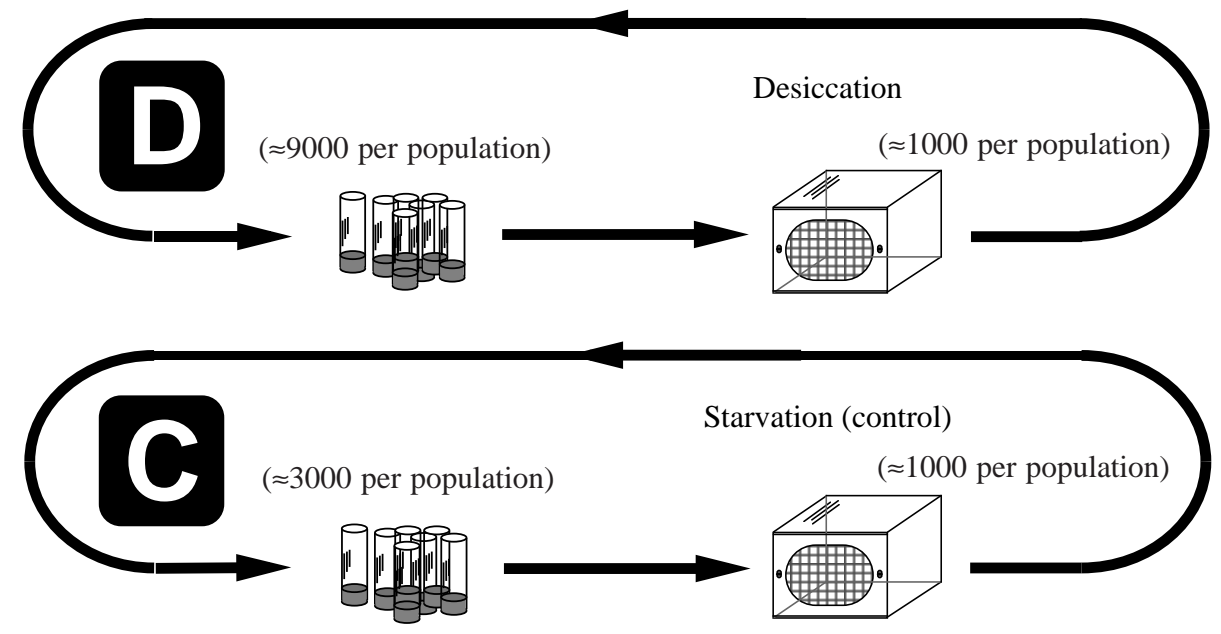

Fig. 1. Schematic illustration of the life cycles for the $\mathrm{C}$ and $\mathrm{D}$ populations. The mild starvation stress experienced by the $\mathrm{C}$ flies serves as a control treatment for the combined starvation and desiccation selection experienced by the D flies.

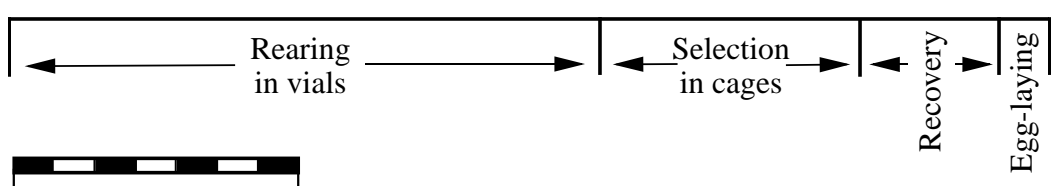

1 week (in days) common 2-week stock cycle with relaxed selection in order to avoid potential effects of the parental environment. Because of the paired nature of the evolutionary history of the flies and the maintenance schedule, assays were performed as paired measurements, i.e. each D population was assayed for water loss measurements or sampled for other analyses along with its corresponding $\mathrm{C}$ control.

\section{Assays for desiccation resistance}

At 4 days post-eclosion, flies were lightly anesthetized with $\mathrm{CO}_{2}$. Ten flies of each sex, from each of the ten D and $\mathrm{C}$ populations, were placed in individual $30 \mathrm{ml}$ vials. A polyethylene sponge was used to keep the flies in the lower half of the vial. Fresh Drierite desiccant (approximately $3 \mathrm{~g}$ ) was added above the sponge, and the vial was sealed with Parafilm. Flies were checked at hourly intervals for death, as indicated by failure to right themselves or to move their legs when their vials were tapped or inverted. Upon death, flies were immediately weighed for determination of tolerance to dehydration (details below).

\section{Water loss rates}

We used a Sable Systems (Henderson, NV, USA) flowthrough respirometry system to measure water loss rates. Dry air was passed at a rate of $25 \mathrm{ml} \mathrm{min}^{-1}$ through $5 \mathrm{ml}$ glass and aluminum chambers containing groups of 20 female flies. Up to eight chambers (including empty control chambers) could be assayed sequentially. The sensor (a modified Li-Cor LI6262 infrared humidity detector) was calibrated by injecting water drops of known volume $(0.5-5 \mu \mathrm{l})$ into the flow stream. The instrument response was linear over this range, with an intercept statistically indistinguishable from zero. Sham injection of air gave no response. Thus, although our measurements were in the $0-0.5 \mu \mathrm{l}$ range, we felt confident in extrapolating from our calibration curve to this range. The entire apparatus was contained in a walk-in temperaturecontrolled room at $25^{\circ} \mathrm{C}$.

Owing to the limitations of our respirometry system, only six sets of 20 flies could be assayed on a given day. Thus, each $\mathrm{C}_{\mathrm{i}}-\mathrm{D}_{\mathrm{i}}$ stock pair was measured, in triplicate, on a different day. Egg collection was staggered so that all flies were 14 days from the egg stage on the day of measurement. For a given set of measurements, eight respirometry chambers were flushed with dry air overnight at $25 \mathrm{ml} \mathrm{min}^{-1}$. Six chambers were used for flies, and two empty chambers served as controls. Twenty female flies per chamber were used in all water loss measurements. Preliminary experiments indicated that water loss rates were relatively high in the first $2 \mathrm{~h}$ following placement in the chambers, then stabilized for the next several hours. We therefore staggered placement of the flies in the respirometer, so that they had been in their chambers for $3 \mathrm{~h}$ before a measurement began. An empty chamber was read first, followed by three chambers containing flies, the second empty chamber, three more experimental chambers, and a repeated measurement of the first empty chamber. D and C flies were alternated during the day, and their overall order (C flies first or D flies first) was alternated from one day to the next. Air flow rates during measurements were $25 \mathrm{ml} \mathrm{min}^{-1}$. Because the chambers were flushed continuously at approximately the same rate before measurement, little water vapor accumulated in the chambers, and washout times were usually short. Even so, we only used data covering approximately the last $30 \mathrm{~min}$ of a $1 \mathrm{~h}$ run.

\section{Cuticular lipid analyses}

Two-week-old flies (approximately 4 days post-eclosion) 
were frozen and stored at $-20^{\circ} \mathrm{C}$ for cuticular lipid analyses. Epicuticular hydrocarbons were isolated as described by Toolson (1982). Ten flies were placed on a silica gel column in a Pasteur pipette, and hydrocarbons were eluted with $6 \mathrm{ml}$ of HPLC-grade hexane. For quantitative determination of cuticular lipid amounts, $2.5 \mu \mathrm{g}$ of $n$-docosane in a small volume of hexane was added to the column along with the flies as an internal standard.

We analyzed cuticular lipids in two ways. Lipid amounts were quantified by capillary gas chromatography of samples containing the $n$-docosane standard, using a $30 \mathrm{~m} \times 0.32 \mu \mathrm{m}$ DB-1 column (J\&W Scientific, Sacramento, CA, USA) in a Hewlett-Packard 5890A gas chromatograph, GC. The chromatograms were also used to investigate differences in cuticular hydrocarbon composition. Hydrocarbons were classified by carbon number (chain length) according to their retention times compared with $n$-alkane standards. Mean chain lengths were computed as the weighted average of the abundance of each component.

Functional differences in the water-proofing abilities of cuticular hydrocarbons depend in part upon their physical properties (Beament, 1945; Toolson et al. 1979; A. G. Gibbs, in preparation). Lipid melting temperatures $\left(T_{\mathrm{m}}\right)$ were determined using Fourier transform infrared (FTIR) spectroscopy, as described by Gibbs and Crowe (1991). This technique is based upon the lipid phase-dependent shift in the infrared absorbance maximum of $-\mathrm{CH}_{2-}$ symmetrical stretching vibrations. Hydrocarbons extracted from groups of ten flies were placed in a temperature-controlled cell holder in a Perkin-Elmer Systems 2000 FTIR spectrometer. The sample temperature was increased in $2-3{ }^{\circ} \mathrm{C}$ increments from $5-10^{\circ} \mathrm{C}$ to $50-60^{\circ} \mathrm{C}$. As indicated by FTIR, hydrocarbons began melting at $20-25^{\circ} \mathrm{C}$ and had finished melting by $45-50^{\circ} \mathrm{C}$. Plots of $-\mathrm{CH}_{2}-$ symmetrical stretching frequency versus temperature were sigmoidal. The midpoint of the phase transition $\left(T_{\mathrm{m}}\right)$ was calculated as the midpoint of a fitted logistic curve.

\section{Water content assays}

Bulk water contents were determined for six groups of five flies from a given sex and population. Flies were frozen in microcentrifuge tubes on dry ice. Within $24 \mathrm{~h}$, each group was weighed on tared pieces of aluminum foil to a precision of $1 \mu \mathrm{g}$ using a Cahn microbalance. The flies were dried overnight at $55^{\circ} \mathrm{C}$ and re-weighed. Water content was calculated as the difference between the wet and dry mass. Preliminary experiments indicated that wet mass did not change for up to $48 \mathrm{~h}$ on dry ice.

Within a few generations of the water content assays, flies were assayed for glycogen and lipid contents, as part of a study of stress and metabolism in these and other Drosophila populations (M. Djawdan, A. K. Chippindale, M. R. Rose and T. J. Bradley, in preparation). Because both energetic substrates can serve as sources of metabolic water (SchmidtNielsen, 1990), we calculated the total amount of metabolic water that could be obtained if these substrates were completely aerobically metabolized (i.e. maximum available metabolic water). Our conversion factors were $0.56 \mathrm{mg} \mathrm{H}_{2} \mathrm{O} \mathrm{mg}^{-1}$ glycogen and $1.07 \mathrm{mg} \mathrm{H}_{2} \mathrm{O} \mathrm{mg}^{-1}$ lipid (Schmidt-Nielsen, 1990). Water bound to glycogen was included in our measurement of bulk water. Glycogen levels were determined using a modified anthrone reaction, and lipid contents were measured by extraction with a Soxhlet apparatus, as in our earlier work (Chippindale et al. 1996).

\section{Dehydration tolerance}

To investigate whether the $\mathrm{C}$ and $\mathrm{D}$ flies differed in their abilities to tolerate water loss, we attempted to measure the water contents of flies as close to the time of death from dehydration as possible. If a fly appeared dead during an hourly check for the desiccation resistance assay (above), it was removed from its vial, and its wet mass was measured immediately. It was then dried overnight at $55^{\circ} \mathrm{C}$, and the water content was determined as described above.

\section{Statistical analyses}

Data were analyzed by mixed-model analyses of variance (ANOVAs) using Systat software. We treated sex and treatment (i.e. C or D selection regime) as fixed effects, with population number (1-5) as a random effect. Populations were treated as $\mathrm{D}_{\mathrm{i}}-\mathrm{C}_{\mathrm{i}}$ pairs during selection and assays, so population effects in statistical analyses are equivalent to block effects. The fundamental unit of replication in these stocks is the population, so graphical data are depicted as the means $( \pm$ S.E.M.) of $N=5$ populations. Different numbers of flies were used in different assays (e.g. individual flies in the dehydration tolerance assay, groups of 20 flies for water loss measurements). For ease of comparison, all measurements have been converted to single fly units, and error estimates have been scaled accordingly.

\section{Results \\ Desiccation resistance}

Flies from desiccation-selected populations survived over twice as long in dry air as their $\mathrm{C}$ controls $(P<0.001$; Fig. 2$)$, with one D female surviving for $84 \mathrm{~h}$. Thus, selection for desiccation resistance has been quite successful, indicating that the original founding $\mathrm{O}$ populations contained substantial genetic variation upon which selection could act. Females survived longer than males $(P<0.001)$. A significant genderby-treatment effect was observed, due to the much greater desiccation resistance of $\mathrm{D}$ females relative to other gender-treatment combinations.

\section{Water loss rates}

Fig. 3 depicts a typical recording for water loss from a $\mathrm{C}$ and D stock pair. For all populations, water loss was characterized by a relatively constant background rate, with intermittent bursts of water loss. We calculated three parameters: (1) total water loss rate, calculated by integrating the area under the curve; (2) 'sporadic' water loss (area of the 


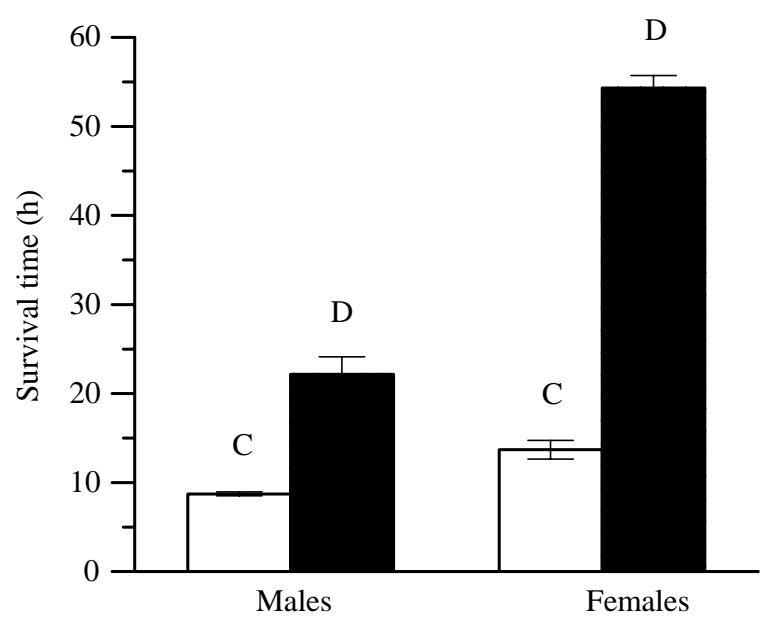

Fig. 2. Mean survival times (desiccation resistance) for individual flies in dry air. For each population (C and D), ten flies of each gender were assayed. Error bars depict standard errors for the means of $N=5$ population means.

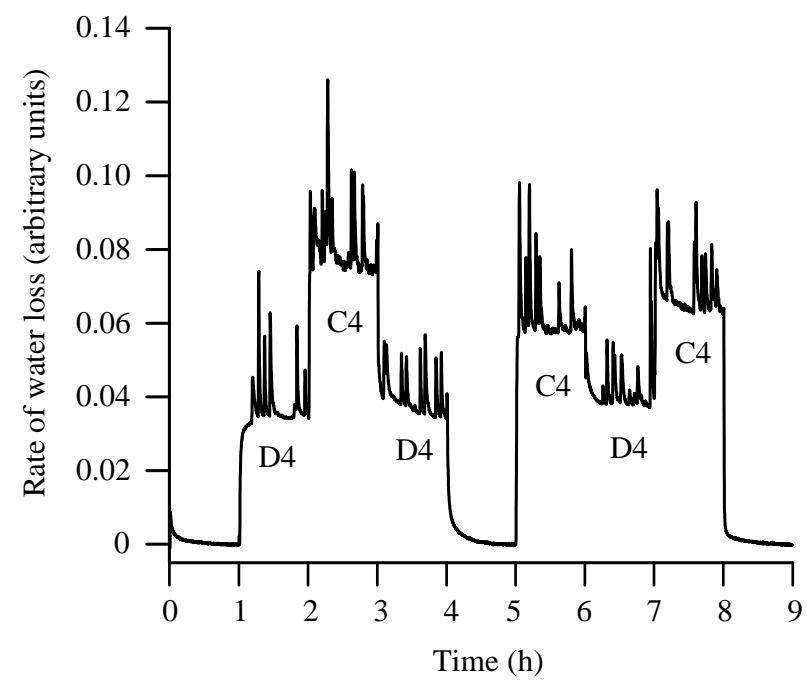

Fig. 3. Representative water loss rate data for control (C) and desiccation-selected (D) flies. The humidity scale was calibrated by injecting known volumes of water and integrating under the area of the resultant peak. The flies in this example were from the $\mathrm{C}_{4}-\mathrm{D}_{4}$ population pair. Each group of 20 females was placed in the respirometer $3 \mathrm{~h}$ before measurement began for their chamber.

peaks); and (3) baseline water loss, calculated as the difference between total and sporadic water loss. Flies from the D populations were larger than $\mathrm{C}$ flies, but this difference was due to their greater water content (see below). Because the $\mathrm{D}$ and $\mathrm{C}$ treatments did not differ in dry mass $(0.444 \pm 0.031 \mathrm{mg}$ and $0.399 \pm 0.024 \mathrm{mg}$ (mean \pm S.E.M.) per fly for $\mathrm{D}$ and $\mathrm{C}$ females, respectively), we have expressed our results on a per fly basis.

Total water loss rates differed significantly between the D and $\mathrm{C}$ stocks. D females lost water at approximately $60 \%$ of the rate for $\mathrm{C}$ females (Fig. $4 ; P<0.001$ ). The difference

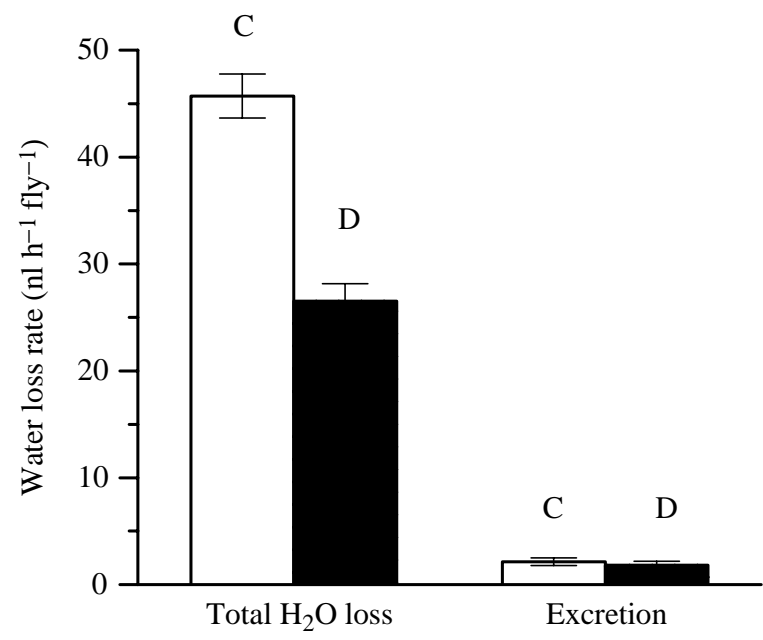

Fig. 4. Water loss rates for control (C) and desiccation-selected (D) female flies. Excretory water loss was calculated from the integrated area of the bursts of water loss shown in Fig. 3. Means and standard errors for $N=5$ populations are provided.

between the stocks was mainly due to differences in the baseline rates of water loss. Rates of 'sporadic' water loss were slightly lower in the $\mathrm{D}$ populations $(P<0.05)$, but still accounted for less than $10 \%$ of total water loss in any given population. On a percentage basis, the $\mathrm{D}$ populations lost relatively more water during these events $(6.7 \%$ of total water loss in the D flies, $4.6 \%$ in the $\mathrm{C}$ flies; $P<0.025)$.

\section{Cuticular lipid analyses}

Eighteen hydrocarbons accounted for approximately $97 \%$ of the total cuticular lipid complement. On the basis of previous analyses of cuticular hydrocarbons from D. melanogaster (Antony and Jallon, 1982; Scott and Jackson, 1988; Graves et al. 1992), these peaks primarily corresponded to alkenes and alkadienes containing 21-31 carbon atoms. Although we did not perform a detailed chemical analysis of cuticular lipid structure, we note that no novel peaks were observed in either $\mathrm{C}$ or D flies. Thus, any differences in cuticular hydrocarbons reflected differences in the distribution of compounds common to both sets of populations, rather than the synthesis of new components.

Flies averaged $1.6 \mu \mathrm{g}$ of total hydrocarbon per fly, and lipid quantity did not differ between genders or between the $\mathrm{C}$ and D populations (Table 1). Lipid melting temperatures $\left(T_{\mathrm{m}}\right)$ differed significantly between genders and treatments (Table 2). Mean $T_{\mathrm{m}}$ values ( \pm S.E.M. for $N=5$ populations) were $36.6 \pm 0.6^{\circ} \mathrm{C}$ and $35.7 \pm 0.5^{\circ} \mathrm{C}$ for $\mathrm{D}$ and $\mathrm{C}$ females, respectively, and $33.9 \pm 0.7^{\circ} \mathrm{C}$ and $30.2 \pm 0.4^{\circ} \mathrm{C}$ for $\mathrm{D}$ and $\mathrm{C}$ males, respectively. An ANOVA of mean hydrocarbon chain length revealed significant gender and treatment differences consistent with the $T_{\mathrm{m}}$ results (Table 3). Surface lipids from desiccation-selected flies were longer, on average, than those of $\mathrm{C}$ flies. When hydrocarbons were grouped by number of carbon atoms (21-31 carbons, odd numbers only), ANOVAs performed on each group separately revealed significant 


\section{A. G. Gibbs, A. K. Chippindale and M. R. Rose}

Table 1. Analysis of variance results for cuticular hydrocarbon quantity in $C$ and $D$ flies

\begin{tabular}{llcccc}
\hline & Sum of & & Mean & \\
Source & squares & d.f. & square & $F$-ratio & 0.2793 \\
Gender & 0.0303 & 1 & 0.0303 & 0.75 & 0.1109 \\
Treatment & 0.6240 & 1 & 0.6240 & 2.4938 & 0.076 \\
Population & 1.4815 & 4 & 0.3704 & 0.1744 \\
Gender $\times$ Treatment & 0.8168 & 1 & 0.8168 & 0.7307 \\
Gender $\times$ Population & 0.4341 & 4 & 0.1085 & 3.7823 \\
Treatment $\times$ Population & 2.2469 & 4 & 0.5617 & 0.6955 \\
Gender $\times$ Treatment $\times$ Population & 0.4131 & 4 & 0.1033 & 0.019 \\
Error & 2.9703 & 20 & 0.1485 &
\end{tabular}

In this and the following tables, gender and treatment (D or C) were treated as fixed effects and population as a random effect.

d.f., degrees of freedom.

Table 2. Analysis of variance results for cuticular lipid melting temperature $\left(\mathrm{T}_{m}\right)$

\begin{tabular}{|c|c|c|c|c|c|}
\hline Source & $\begin{array}{l}\text { Sum of } \\
\text { squares }\end{array}$ & d.f. & $\begin{array}{l}\text { Mean } \\
\text { square }\end{array}$ & $F$-ratio & $P$ \\
\hline Gender & 260.2456 & 1 & 260.2456 & 79.8912 & 0.001 \\
\hline Treatment & 81.9250 & 1 & 81.9250 & 48.4047 & 0.005 \\
\hline Population & 33.9155 & 4 & 8.4789 & 1.8301 & 0.14 \\
\hline Gender $\times$ Treatment & 30.6170 & 1 & 30.6170 & 4.2775 & 0.25 \\
\hline Gender $\times$ Population & 13.0299 & 4 & 3.2575 & 0.7031 & 0.59 \\
\hline Treatment $\times$ Population & 6.7701 & 4 & 1.6925 & 0.3653 & 0.83 \\
\hline Gender $\times$ Treatment $\times$ Population & 28.6309 & 4 & 7.1577 & 1.5450 & 0.25 \\
\hline Error & 203.8488 & 44 & 4.6329 & & \\
\hline
\end{tabular}

d.f., degrees of freedom.

Table 3. Analysis of variance results for mean cuticular hydrocarbon chain length

\begin{tabular}{|c|c|c|c|c|c|}
\hline Source & $\begin{array}{l}\text { Sum of } \\
\text { squares }\end{array}$ & d.f. & $\begin{array}{l}\text { Mean } \\
\text { square }\end{array}$ & $F$-ratio & $P$ \\
\hline Gender & 21.7436 & 1 & 21.7436 & 259.4079 & 0.001 \\
\hline Treatment & 1.5031 & 1 & 1.5031 & 16.6858 & 0.025 \\
\hline Population & 0.7928 & 4 & 0.1982 & 2.4474 & 0.08 \\
\hline Gender $\times$ Treatment & 0.0221 & 1 & 0.0221 & 0.1447 & 0.75 \\
\hline Gender $\times$ Population & 0.3353 & 4 & 0.0838 & 1.0351 & 0.41 \\
\hline Treatment $\times$ Population & 0.3603 & 4 & 0.0901 & 1.1124 & 0.38 \\
\hline Gender $\times$ Treatment $\times$ Population & 0.6111 & 4 & 0.1528 & 1.8864 & 0.15 \\
\hline Error & 1.6196 & 20 & 0.0810 & & \\
\hline
\end{tabular}

d.f., degrees of freedom.

gender differences for each chain length. Males had relatively greater amounts of 21-, 23- and 25-carbon hydrocarbons, whereas females had relatively higher levels of the 27-, 29- and 31-carbon species (Fig. 5; $P<0.05$ ). Four lipid groups exhibited significant differences between the $\mathrm{D}$ and $\mathrm{C}$ selection treatments $(\mathrm{C}>\mathrm{D}$ for 21 - and 23-carbon lipids, $\mathrm{D}>\mathrm{C}$ for 25 - and 29 -carbon species; $P<0.05)$. A significant gender-by-treatment interaction was observed for hydrocarbons containing 25 carbon atoms.
Water content: bulk water and metabolic water

On a wet mass basis, female flies weighed more than males, and D flies weighed more than $\mathrm{C}$ flies $(P<0.01$; Table 4$)$. The selection-related difference in mass was due to the higher water content of the desiccation-selected populations, since dry mass did not differ significantly between $\mathrm{D}$ and $\mathrm{C}$ populations. Individual female D flies averaged $241 \mu \mathrm{g}$ more water than $\mathrm{C}$ females (Fig. 6). D males also contained over $20 \%$ more water than $\mathrm{C}$ males (results not shown). 


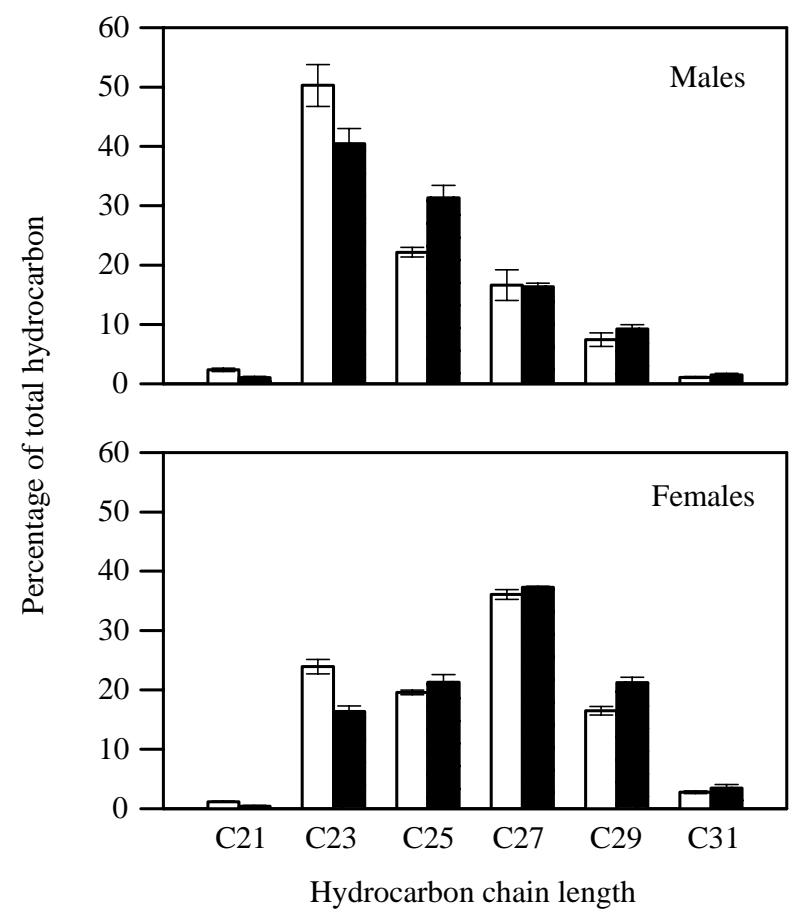

Fig. 5. Effects of selection treatment and gender upon chain lengths of cuticular hydrocarbons. Data for C23-C31 species include 2-5 different GC peaks. Open bars, C populations; filled bars, D populations. Values are means \pm S.E.M. for $N=5$ populations.

Six generations after these measurements, glycogen levels and total lipid contents were determined by M. Djawdan, A. K. Chippindale, M. R. Rose and T. J. Bradley (in preparation). Because these substances can be used as a source of metabolic water, we used their data to calculate the potential metabolic water that could be produced from each substrate. As observed previously (Graves et al. 1992), the D flies contained significantly more glycogen than the $\mathrm{C}$ flies and, therefore, could obtain more metabolic water from glycogen metabolism. However, the $\mathrm{C}$ flies contained significantly more lipid. When total available metabolic water from glycogen and lipid was combined, both $\mathrm{D}$ and $\mathrm{C}$ flies had nearly identical amounts (approximately $154 \mu \mathrm{g}$ per female fly, Fig. 6).

Table 4. Body sizes of desiccation-selected and control populations

\begin{tabular}{lcc}
\hline Population & $\begin{array}{c}\text { Wet mass per fly } \\
(\mathrm{mg})\end{array}$ & $\begin{array}{c}\text { Dry mass per fly } \\
(\mathrm{mg})\end{array}$ \\
\hline D females & $1.444 \pm 0.102$ & $0.444 \pm 0.031$ \\
D males & $0.851 \pm 0.070$ & $0.253 \pm 0.016$ \\
C females & $1.158 \pm 0.019$ & $0.399 \pm 0.024$ \\
C males & $0.731 \pm 0.013$ & $0.248 \pm 0.009$
\end{tabular}

Data are means \pm S.E.M. for $N=5$ populations, with six groups of five flies each measured per population.

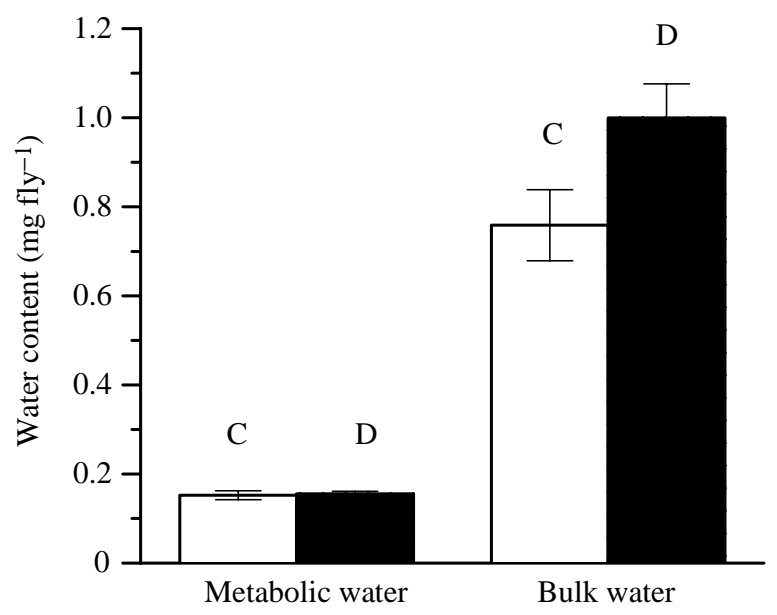

Fig. 6. Water reserves for control (C) and desiccation-selected (D) females. Bulk water was calculated from the difference between wet and dry mass, and metabolic water was calculated from data in $\mathbf{M}$. Djawdan and others (in preparation). Values are means \pm S.E.M. for $N=5$ populations.

\section{Dehydration tolerance}

Dehydration tolerance, as indicated by water content at death, can be quantified in two ways: either as absolute water content or as percentage water. On a percentage basis, C flies actually appeared to tolerate greater dehydration (45\% body water at death versus $56 \%$ for $\mathrm{D}$ flies, Fig. 7A). This counterintuitive result was due to the fact that the $\mathrm{C}$ flies had a greater dry mass at death $(P<0.001$, Fig. $7 \mathrm{~B})$, but the $\mathrm{D}$ and $\mathrm{C}$ flies did not differ from each other in water content at time of death $(P>0.25$, Fig. $7 \mathrm{C})$. Females contained more water when they died than males $(P<0.001)$ owing to their larger overall size. Since absolute water contents were the same, but the $\mathrm{C}$ flies had a greater dry mass, percentage water content at death was greater in $\mathrm{D}$ flies.

\section{Discussion}

The $\mathrm{D}$ and C populations were founded in 1988 from the Ostocks, which at that time survived desiccation for approximately $15 \mathrm{~h}$ (for females; males survived approximately $9 \mathrm{~h}$ ). The $\mathrm{D}$ flies have since diverged dramatically from their $\mathrm{O}$ ancestors and their unselected controls; desiccation survival times for $\mathrm{D}$ females now average over 2 days (Fig. 2). At the organismal level, there are only three possible, non-exclusive mechanisms for increased desiccation resistance: flies can contain more water, they can restrict its loss or they can tolerate loss of a greater amount of water. Below we consider the impact of each of these three mechanisms.

\section{Water loss rates}

Comparative studies have shown that Drosophila species and populations from apparently xeric habitats lose water more slowly and survive desiccation stress longer than those from mesic environments (Eckstrand and Richardson, 1980, 1981; 

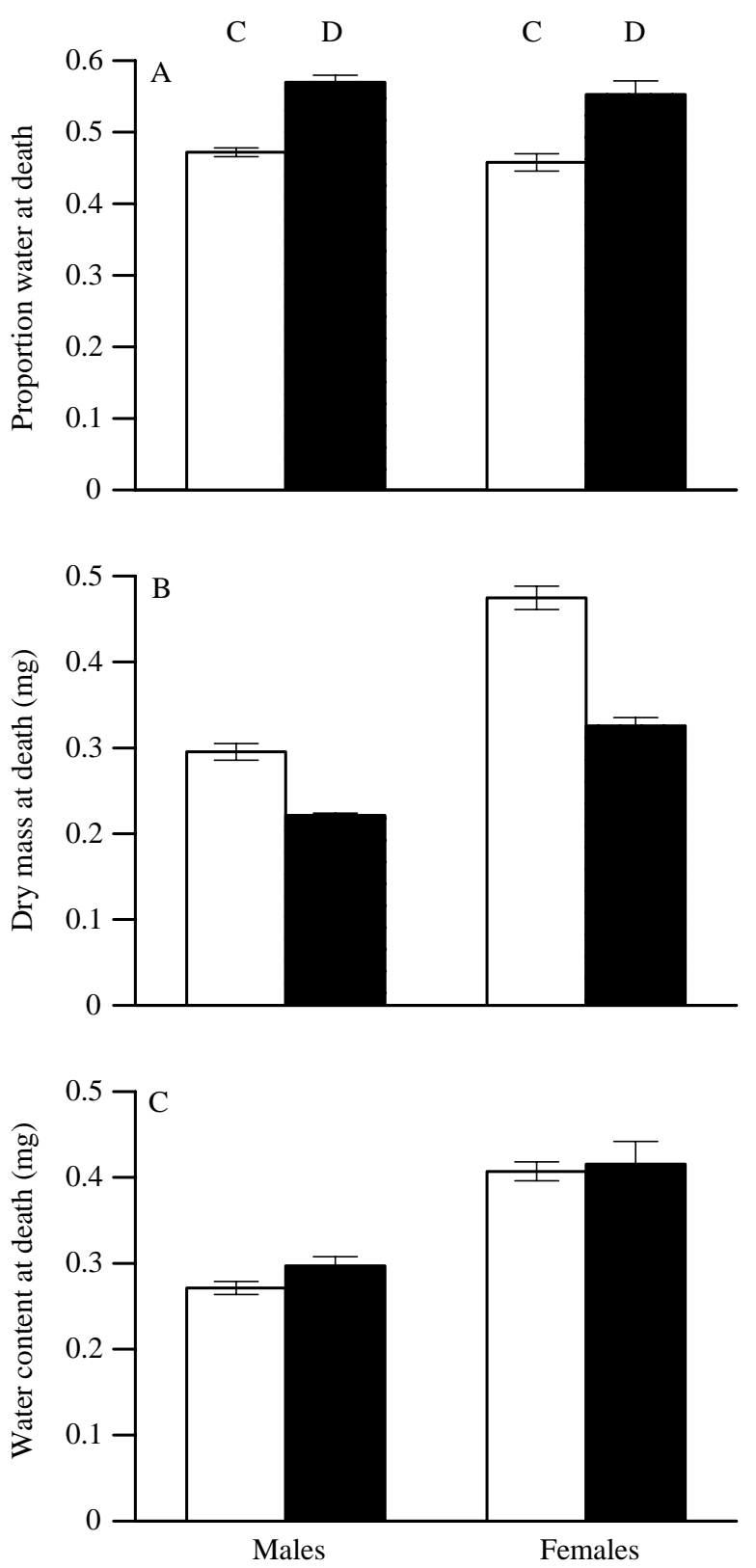

Fig. 7. Analysis of dehydration tolerance for control (C) and desiccation-selected (D) flies. Wet and dry mass for individual flies were determined immediately after their apparent death. The proportion composed of water was calculated as the water content divided by the wet mass. Means and standard errors for $N=5$ populations are shown.

Hoffmann, 1991; A. G. Gibbs and L. M. Matzkin, unpublished observations). In our laboratory-evolved populations, we found that desiccation-selected female flies lost water approximately $60 \%$ as rapidly as their controls. Several mechanisms can be envisioned to account for these differences. Cuticular permeability could be reduced, respiratory water loss could be lowered by decreasing metabolic rate or regulating spiracular opening more tightly, or excretory water loss could be reduced.

Both C and D flies exhibited bursts of water loss at irregular intervals. On the basis of measurements of $\mathrm{CO}_{2}$ release from individual flies (Williams et al. 1997), we do not believe these represented respiratory water loss associated with spiracular opening during discontinuous ventilation (Hadley 1994a,b). Instead, the bursts probably reflected excretory events, such as defecation. The D females lost slightly less water overall during these events than the $\mathrm{C}$ females. However, water loss by this route accounted for less than $10 \%$ of total loss in any given population and, on a percentage basis, was higher in the D populations. Thus, it appears that reduction of excretory water loss is not a major contributor to desiccation resistance in the D flies.

Since presumed excretory water loss accounted for less than $10 \%$ of the total, over $90 \%$ of water loss could be attributed to differences in baseline rates. This difference included both respiratory and cuticular components, and our techniques were unable to distinguish between these routes. As noted above, ventilatory patterns may differ between $\mathrm{D}$ and $\mathrm{C}$ flies, although overall metabolic rates do not differ and are not affected by dehydration stress (Djawdan et al. 1997). Thus, it is unclear to what extent differences in respiratory water loss contribute to increased desiccation resistance. In several other insect species, cuticular water loss has been shown to comprise over $90 \%$ of total water loss (Hadley, 1994a,b), equivalent to the basal contribution in our flies. Other insects that have been studied have been much larger than Drosophila species, and it is not known how differences in body size or taxon can affect the relative importance of respiratory water loss. However, available data suggest that lower water loss rates in the D flies may result largely from reduced cuticular permeability.

If this is case, it is reasonable that cuticular lipids might be responsible, since these form the primary passive barrier to evaporative water loss. Two aspects of cuticular lipids may be important: lipid amount and lipid composition. Graves et al. (1992) found no differences in cuticular hydrocarbon quantities or composition between the aging-selected $\mathrm{O}$ populations and their short-lived controls, despite differences in desiccation resistance and in water loss rates of dead flies. We found no significant differences between C and D flies in the quantity of cuticular hydrocarbons, indicating that desiccation-selected flies have not reduced water loss rates by increasing the thickness of the waterproofing layer. Instead, changes in surface lipid composition seem to have played a greater role in the evolution of desiccation resistance.

The mechanistic link between lipid composition and water loss rates is believed to be the physical properties of the cuticular lipids (Beament, 1945; Noble-Nesbitt, 1991). Hydrocarbons with longer chain-lengths would be expected to melt at higher temperatures and therefore provide a better barrier to water loss. We found that D flies had longer chainlength cuticular hydrocarbons than control populations, and that lipid melting temperatures were higher than those of $\mathrm{C}$ flies. The difference in mean hydrocarbon chain length, approximately 0.5 carbons per molecule, was consistent with the observed increase in $T_{\mathrm{m}}$ values of approximately $1{ }^{\circ} \mathrm{C}$ (Gibbs and Pomonis, 1995). We note that the lipid melting 
points we measured $\left(>35^{\circ} \mathrm{C}\right.$ for females) were above the longterm (>15 years, $>300$ generations) thermal regime experienced by the flies $\left(25^{\circ} \mathrm{C}\right)$ since their ancestors were originally collected. However, most lipid samples began to melt below $25^{\circ} \mathrm{C}$, so that detectable changes in phase behavior were apparent at the flies' normal temperature. It is not known how partial lipid melting would affect cuticular permeability.

\section{Water content}

Besides reductions in water loss rates, desiccation resistance can be enhanced by having more water to start with. Graves et al. (1992) found that the D populations contained higher levels of glycogen than the $\mathrm{C}$ populations and suggested that glycogen serves as a source of metabolic water during desiccation. However, a rough estimate of the potential contribution of glycogen to desiccation resistance in the D flies indicates the situation is not so simple. M. Djawdan, A. K. Chippindale, M. R. Rose and T. J. Bradley, in preparation), in experiments performed shortly after ours, found that $\mathrm{D}$ females averaged $0.118 \mathrm{mg}$ glycogen per individual, and we measured water loss rates of $0.0265 \mu \mathrm{h} \mathrm{h}^{-1} \mathrm{fly}^{-1}$ for $\mathrm{D}$ females. Assuming that metabolism of $1 \mathrm{~g}$ of glycogen results in the production of $0.56 \mathrm{~g}$ of water (Schmidt-Nielsen, 1990), metabolic water production from glycogen could offset water loss during desiccation stress for only $0.56 \times 0.118 / 0.0265=2.5 \mathrm{~h}$. An additional complication is that the $\mathrm{C}$ flies have greater lipid stores than the $\mathrm{D}$ flies, sufficient to give both sets of populations equivalent total metabolic water stores (Fig. 6). We note that the $\mathrm{C}$ populations are subjected to mild starvation selection, and that flies subjected to strong starvation selection have even greater lipid stores (Chippindale et al. 1996).

The largest store of water in the body is water itself. We found that D flies contained approximately $30 \%$ more water than the $\mathrm{C}$ flies (Fig. 6). In fact, the difference in water content between $\mathrm{C}$ and $\mathrm{D}$ flies was greater than the combined available metabolic water from both energetic substrates. This finding further demonstrates that glycogen is at best a minor source of water in the D flies. Thus, higher glycogen levels in the D flies must be due to some other factor. One possibility is that glycogen serves as a metabolic fuel during desiccation stress. In accordance with this idea, Djawdan et al. (1997) observed that D. melanogaster subjected to desiccation stress primarily metabolize carbohydrates, whereas starvation-stressed flies use lipids. It should also be noted that glycogen may bind 3-5 times its mass in water (Schmidt-Nielsen, 1990). If glycogen binds $4 \mathrm{~g}$ water $\mathrm{g}^{-1}$, carbohydrate stores in the $\mathrm{D}$ females could bind approximately $47 \%$ of the total water content. Thus, glycogen may act as a 'sponge' to store water. This water would become available only as glycogen was metabolized; thus, shifting of metabolic fuels during desiccation to glycogen (Djawdan et al. 1997) would facilitate the release of water of hydration.

Blows and Hoffmann (1993), using desiccation-selected populations of $D$. serrata, found no differences in glycogen or lipid content, and none was evident for total water content (calculated from Table 5 in Blows and Hoffmann, 1993).
Instead, they observed that metabolic rates increased at low humidity, but to a lesser extent for desiccation-selected lines. Blows and Hoffmann (1993) suggested that reduced respiratory water loss was responsible for increased desiccation resistance. For the $\mathrm{C}$ and $\mathrm{D}$ populations of $D$. melanogaster used in this study, Djawdan et al. (1997) found instead that metabolic rates were unaffected by desiccation stress and did not differ between selected and control flies.

The conflicting results obtained in these two studies might be explained by differences in species or number of generations of selection [14 for Blows and Hoffmann (1993); approximately 120 for Djawdan et al. (1997) and this study]. In addition, we feel that the differences in metabolic rates seen by Blows and Hoffmann (1993) may have been an experimental artefact, and we offer an alternative explanation. Blows and Hoffmann (1993) measured metabolic rates under humid conditions in a Gilson respirometer, by using $2.5 \mathrm{~mol} \mathrm{l}^{-1}$ $\mathrm{NaOH}$ to absorb $\mathrm{CO}_{2}$. 'Dry' conditions were provided by using solid $\mathrm{NaOH}$ instead. Sodium hydroxide is very hygroscopic, so that water lost by the flies under dry conditions could have been absorbed by the $\mathrm{NaOH}$ pellets and interpreted as $\mathrm{CO}_{2}$ production. Thus, increased metabolic rates at low humidity could actually reflect increased net water loss under these conditions (Cooper, 1983), and the apparent difference in metabolic rates in dry air could have been partly due to reduced water loss in desiccation-selected lines. We suggest that the experiments by Blows and Hoffmann (1993) could not distinguish between reduced metabolic rates or water loss rates in desiccation-selected flies, and their results and those observed for the Rose laboratory populations may not be inconsistent.

\section{Dehydration tolerance}

The third physiological component of desiccation resistance is the ability to tolerate water loss. A survey of the literature on insect dehydration tolerance by Hadley (1994a) found widely varying estimates for measures such as percentage water content at death and percentage of total body water which an insect can lose. We found that the absolute water content at death did not differ between the $\mathrm{C}$ and $\mathrm{D}$ flies (Fig. 7C). However, owing to their continued metabolism over a much longer period of desiccation stress, the D flies had a significantly lower dry mass at death. (As noted above, dry mass did not differ between hydrated $\mathrm{C}$ and $\mathrm{D}$ flies.) This result led to the counterintuitive finding that $\mathrm{C}$ flies could tolerate greater dehydration, as measured by percentage body water at time of death (Fig. 7A).

The lack of differentiation in dehydration tolerance indicates that the D populations have not taken advantage of all possible mechanisms of desiccation resistance. Several possible explanations are available. The first is that little or no genetic variation in dehydration tolerance existed in the original founding populations, and that no new mutations having significant effects on dehydration tolerance have arisen and spread in the more than 100 generations since the $\mathrm{C}$ and $\mathrm{D}$ populations were founded. Another possibility is that the 


\section{A. G. Gibbs, A. K. Chippindale and M. R. Rose}

selection differential involving dehydration tolerance has been too small for significant differences to have appeared yet. A third alternative is that selection has acted upon the dehydration threshold, but that genetic correlations with other characters or pleiotropic effects at other life stages have acted in the opposite direction, so that no net change has occurred. A physiological explanation is that there may be an absolute limit to how little water a fly can have and remain viable. Insects may need a certain amount of hemolymph for nutrient and hormone transport, and cells may need a certain amount of water to remain viable. Dehydration tolerance may represent a physiological constraint which cannot be improved without evolving novel traits such as those exhibited by anhydrobiotes (Crowe et al. 1992). Our experiments were not designed to distinguish among these alternatives.

\section{Contributions of traits to evolved desiccation resistance}

Under desiccating conditions in vials (i.e. still air), D females survived for approximately $52 \mathrm{~h}$, whereas $\mathrm{C}$ females survived for only $15 \mathrm{~h}$ (Fig. 2). Since we have a complete water budget, at the organismal level, for the $\mathrm{D}$ and $\mathrm{C}$ populations, it is possible to assess the relative importance of different mechanisms for desiccation resistance. We can do so by quantifying how many hours of additional desiccation resistance are conferred by reduced water loss, increased water content or differences in dehydration tolerance. Only the case for female flies will be considered, because females survive desiccation stress much longer than males (Fig. 2) and we have more complete data for females. In addition, female flies appear to be under stronger selection for survival of desiccation, because few males survive the selection procedure, as discussed below.

Since differences in dehydration tolerance (as indicated by water content at death) have not appeared during the evolution of desiccation resistance (Fig. 7), reduction of water loss and increased water content must be sufficient to explain the greater desiccation resistance of the $\mathrm{D}$ flies. If one assumes that water loss rates measured for groups of 20 flies after $3 \mathrm{~h}$ in a flow-through respirometer are an accurate index of water loss rates for individual flies during desiccation in still air, then the reduced water loss rates in the D flies (approximately $60 \%$ of the rate for the $\mathrm{C}$ flies) will result in survival times of $15 \mathrm{~h} / 0.6=25 \mathrm{~h}$. Thus, reduction of water loss rates alone has increased desiccation resistance of the D flies by $10 \mathrm{~h}$.

The greater water content in $\mathrm{D}$ females, which will be lost at a lower rate, can account for $0.241 \mathrm{mg} \mathrm{H} 2 \mathrm{O} / 0.0265 \mathrm{mg} \mathrm{H} 2 \mathrm{O}$ lost per hour $=9.1 \mathrm{~h}$. Metabolic water contents of the D and C flies were not significantly different (Fig. 6). However, the greater desiccation resistance of the D flies implies (in the absence of differences in metabolic rates; Djawdan et al. 1997) that the $\mathrm{D}$ flies will generate more metabolic water than the $\mathrm{C}$ flies before death. D females contained energy stores sufficient to generate $0.156 \mathrm{mg}$ of metabolic water. This sets an upper limit of $0.156 / 0.0265=5.9 \mathrm{~h}$ of desiccation resistance available from metabolic water.

On the basis of these calculations, reduced water loss rates and increased water content could account for approximately 25 of the $37 \mathrm{~h}$ of increased desiccation resistance in $\mathrm{D}$ females. Several factors may contribute to the discrepancy between these values. Water loss rates may be lower under the conditions of still air found during desiccation assays, so these calculations could underestimate the contributions of both reduced water loss rates and increased water content. In addition, water loss rates progressively decline by up to $20 \%$ during longer periods in the respirometer (A. G. Gibbs, unpublished results), which will further extend desiccation resistance. Finally, although every effort was taken to standardize conditions, environmental differences between experimental generations could also have affected our results.

An interesting aspect of the gender-specific differences in desiccation resistance is that males rarely survive the selection treatment. It appears that desiccation selection has had differing effects upon males and females, with $\mathrm{D}$ males being selected for early adult mating success rather than desiccation resistance (A. K. Chippindale, A. G. Gibbs and M. R. Rose, in preparation). Since no mating is observed during or after selection (A. K. Chippindale, unpublished observations), female D flies must mate early, survive desiccation and lay eggs afterwards. Despite the differing selection pressures upon males and females, both sexes exhibited increased resistance to desiccation, using similar physiological mechanisms (e.g. greater water content), presumably because they share most of their genetic material. Similar sex-specific responses to selection have also been observed in starvation-selected populations (Chippindale et al. 1996).

\section{Conclusions}

The D and C populations provide an especially straightforward example of the utility of experimental evolution studies as a complementary approach to comparative studies. The selective force is known: desiccation survival at 14 days past the egg stage. Each D population is paired with a control $\mathrm{C}$ population, which is treated in as identical a manner as possible, except for the lack of water. Large population sizes are maintained, minimizing artifacts associated with genetic drift or founder effects. The replicated structure of the populations provides statistical power unattainable in two-species comparisons (Garland and Adolph, 1994). These populations constitute a fivefold replicated experimental equivalent of the two-species comparison, with the additional benefit of known selection histories. Finally, insect water balance has been widely studied (Hadley, 1994a), so that the prospect exists for understanding the evolution of this physiological system at whatever level of organization and depth of detail one wants.

The D populations have responded to desiccation selection by reducing water loss, possibly via differences in cuticular lipid properties. They have a higher water content, but overall metabolic water stores do not differ between $\mathrm{D}$ and $\mathrm{C}$ populations, and contribute less than $6 \mathrm{~h}$ to desiccation resistance. Dehydration tolerance does not differ between D and $\mathrm{C}$ populations, in spite of more than 100 generations under conditions which would appear to favor differentiation. Thus, 
the $\mathrm{D}$ populations have evolved increased desiccation resistance by only some of the possible physiological mechanisms. It remains to be seen whether the lack of differentiation in metabolic water content or dehydration tolerance is due to the lack of genetic variation for these characters, to genetic or physiological constraints, or to other factors.

We thank A. Tabatabaee for assistance with water loss measurements, R. Begino for help with cuticular lipid analyses, D. Nghiem for help with desiccation resistance and dehydration tolerance assays, M. Djawdan and A. E. Williams for technical advice and access to unpublished data, J. B. Lighton for assistance with trouble-shooting the respirometer, T. J. Nusbaum and L. D. Mueller for statistical advice, and V. A. Pierce, A. F. Bennett and T. J. Bradley for helpful discussions and comments on the manuscript. The D and C populations were maintained by L. N. Vu and M. Sheik. This research was funded by a Medical Education and Research Society Young Investigator Award and NSF grant IBN9317471 to A.G.G. and NIH grant AG09970 to M.R.R.

\section{References}

Antony, C. And JaLlon, J. (1982). The chemical basis for sex recognition in Drosophila melanogaster. J. Insect Physiol. 28, 873-880.

BeAment, J. W. L. (1945). The cuticular lipoids of insects. J. exp. Biol. 21, 115-131.

Bennett, A. F., Dao, K. M. and Lenski, R. E. (1990). Rapid evolution in response to high-temperature selection. Nature $\mathbf{3 4 6}$, 79-81.

Blows, M. W. And Hoffmann, A. A. (1993). The genetics of central and marginal populations of Drosophila serrata. I. Genetic variation for stress resistance and species borders. Evolution 47, $1255-1270$.

Chippindale, A. K., Chu, T. J. And Rose, M. R. (1996). Complex trade-offs and the evolution of starvation resistance in Drosophila melanogaster. Evolution 50, 753-766.

COOPER, P. D. (1983). Components of evaporative water loss in the desert tenebrionid beetles Eleodes armata and Cryptoglossa verrucosa. Physiol. Zool. 56, 47-55.

Crow, J. F. (1957). Genetics of insect resistance to chemicals. A. Rev. Ent. 2, 227-246.

Crowe, J. H., Hoekstra, F. A. And Crowe, L. M. (1992). Anhydrobiosis. A. Rev. Physiol. 54, 579-599.

Djawdan, M., Rose, M. R. AND BradLey, T. J. (1997). Does selection for stress resistance lower metabolic rate? Ecology (in press).

ECKSTRAND, I. A. AND RichaRdSON, R. H. (1980). Comparison of some water balance characteristics in several Drosophila species which differ in habitat. Env. Ent. 9, 716-720.

ECKSTRAND, I. A. AND RICHARDSON, R. H. (1981). Relationships between water balance properties and habitat characteristics in the sibling Hawaiian drosophilids, D. mimica and D. kambysellisi. Oecologia 50, 337-341.

Endler, J. A. (1986). Natural Selection in the Wild. Princeton, NJ: Princeton University Press.

Garland, T. AND AdolPh, S. (1994). Why not to do two-species comparative studies: limitations on inferring adaptation. Physiol. Zool. 67, 797-828.
Garland, T. And Carter, P. A. (1994). Evolutionary physiology. A. Rev. Physiol. 56, 579-621.

GibBS, A. AND CROwe, J. H. (1991). Intra-individual variation in cuticular lipids studied using Fourier transform infrared spectroscopy. J. Insect Physiol. 37, 743-748.

GibBS, A. AND Pomonis, J. G. (1995). Physical properties of insect cuticular hydrocarbons: the effects of chain length, methylbranching and unsaturation. Comp. Biochem. Physiol. B 112, 243-249.

Graves, J. L., Toolson, E. C., Jeong, C., Vu, L. N. And Rose, M. R. (1992). Desiccation, flight, glycogen and postponed senescence in Drosophila melanogaster. Physiol. Zool. 65, 268-286.

Gupta, K., LeE, Y. And Yin, J. (1995). Extremo-phage: In vitro selection of tolerance to a hostile environment. J. molec. Evol. 41, 113-114.

Hadley, N. F. (1994a). Water Relations of Terrestrial Arthropods. San Diego: Academic Press.

HadLey, N. F. (1994b). Ventilatory patterns and respiratory transpiration in adult terrestrial insects. Physiol. Zool. 67, 150-189.

Harvey, P. H. AND Pagel, M. D. (1991). The Comparative Method in Evolutionary Biology. Oxford: Oxford University Press. 239pp.

Hoffmann, A. A. (1991). Acclimation for desiccation resistance in Drosophila: Species and population comparisons. J. Insect Physiol. 37, 757-762.

Hoffmann, A. A. And Parsons, P. A. (1989). An integrated approach to environmental stress tolerance and life history variation: desiccation tolerance in Drosophila. Biol. J. Linn. Soc. 37, 117-136.

Hoffmann, A. A. And Parsons, P. A. (1991). Evolutionary Genetics and Environmental Stress. Oxford: Oxford University Press. 284pp.

Huey, R. B., Partridge, L. And Fowler, K. (1991). Thermal sensitivity of Drosophila melanogaster responds rapidly to laboratory natural selection. Evolution 45, 751-756.

Joshi, A., Knight, C. D. AND Mueller, L. D. (1996). Genetics of larval urea tolerance in Drosophila melanogaster. Heredity $\mathbf{7 7}$, 33-39.

Lauder, G. V., Leroi, A. M. And Rose, M. R. (1993). Adaptations and history. Trends Ecol. Evol. 8, 294-297.

LENSKI, R. E. AND BenNetT, A. F. (1993). Evolutionary response of Escherichia coli to thermal stress. Am. Nat. 142, S47-S64.

Leroi, A. M., Rose, M. R. And Lauder, G. V. (1994). What does the comparative method reveal about adaptation? Am. Nat. 143, 381-402.

McKenzie, J. A. And Clarke, G. M. (1988). Diazonin resistance, fluctuating asymmetry and fitness in the Australian sheep blow fly, Lucilia cuprina. Genetics 120, 213-220.

Noble-Nesbitt, J. (1991). Cuticular permeability and its control. In Physiology of the Insect Epidermis (ed. K. Binnington and A. Retnakaran), pp. 252-283. East Melbourne, Victoria, Australia: CSIRO Publications.

Rose, M. R. (1984). Laboratory evolution of postponed senescence in Drosophila melanogaster. Evolution 38, 1004-1010.

Rose, M. R., Graves, J. L. And Hutchison, E. W. (1990). The use of selection to probe patterns of pleiotropy in fitness characters. In Insect Life Cycles: Genetics, Evolution and Co-ordination (ed. F. Gilbert), pp. 29-42. New York: Springer-Verlag.

Rose, M. R., Nusbaum, T. J. and Chippindale, A. K. (1996). Laboratory evolution: the experimental wonderland and the Cheshire cat syndrome. In Adaptation (ed. M. R. Rose and G. V. Lauder), pp. 221-241. San Diego, CA: Academic Press.

Rose, M. R., Vu, L. N., Park, S. U. and Graves, J. L. (1992). 


\section{A. G. Gibbs, A. K. Chippindale and M. R. Rose}

Selection on stress resistance increases longevity in Drosophila melanogaster. Exp. Gerontol. 27, 241-250.

Schmidt-Nielsen, K. (1990). Animal Physiology: Adaptation and Environment. 4th edn. Cambridge: Cambridge University Press. 602pp.

SCOTT, D. AND JACKSON, L. L. (1988). Interstrain comparison of malepredominant antiaphrodisiacs in Drosophila melanogaster. J. Insect Physiol. 34, 863-871.

Service, P. M., Hutchinson, E. W., MacKinley, M. D. and Rose, M. R. (1985). Resistance to environmental stress in Drosophila melanogaster selected for postponed senescence. Physiol. Zool. 58, 380-389.

Service, P. M., Hutchison, E. W. And Rose, M. R. (1988). Multiple genetic mechanisms for the evolution of senescence in Drosophila melanogaster. Evolution 42, 708-716.

Toolson, E. C. (1982). Effects of rearing temperature on cuticle permeability and epicuticular lipid composition in Drosophila pseudoobscura. J. exp. Zool. 222, 249-253.

Toolson, E. C., White, T. R. And Glaunsinger, W. S. (1979). Electron paramagnetic resonance spectroscopy of spin-labelled cuticle of Centruroides sculpturatus (Scorpiones: Buthidae): correlation with thermal effects on cuticular permeability. J. Insect Physiol. 25, 271-275.

Williams, A. E., Rose, M. R. AND Bradley, T. J. (1997). CO$_{2}$ release patterns in Drosophila melanogaster: the effect of selection for desiccation resistance. J. exp. Biol. 200, 615-624. 